\title{
Targeting TSH and IGF-1 Receptors to Treat Thyroid Eye Disease
}

\author{
Susanne Neumann Christine C. Krieger Marvin C. Gershengorn \\ Laboratory of Endocrinology and Receptor Biology, National Institute of Diabetes and Digestive and \\ Kidney Diseases, National Institutes of Health Bethesda, Bethesda, MD, USA
}

\section{Keywords}

Thyroid-stimulating hormone receptor $\cdot$ Insulin-like growth factor 1 receptor . Crosstalk - Antagonists - Thyroid eye disease

\begin{abstract}
Graves' disease (GD) is an autoimmune disease caused in part by thyroid-stimulating antibodies (TSAbs) that activate the thyroid-stimulating hormone receptor (TSHR). In Graves' hyperthyroidism (GH), TSAbs cause persistent stimulation of thyroid cells leading to continuous thyroid hormone synthesis and secretion. Thyroid eye disease (TED), also called Graves' orbitopathy, is an orbital manifestation of GD. We review the important roles of the TSHR and the insulin-like growth factor 1 receptor (IGF-1R) in the pathogenesis of TED and discuss a model of TSHR/IGF-1R crosstalk that considers two pathways initiated by TSAb activation of TSHR in the eye, an IGF-1R-independent and an IGF-1R-dependent signaling pathway leading to hyaluronan (HA) secretion in orbital fibroblasts. We discuss current and future therapeutic approaches targeting the IGF-1R and TSHR. Teprotumumab, a human monoclonal anti-IGF-1R-blocking antibody, has been approved as an effective treatment in patients with
\end{abstract}

karger@karger.com www.karger.com/etj

Karger"
TED. However, as the TSHR seems to be the primary target for TSAbs in patients with GD, future therapeutic interventions directly targeting the TSHR, e.g. blocking antibodies and small molecule antagonists, are being developed and have the advantage to inhibit the IGF-1R-independent as well as the IGF-1R-dependent component of TSAb-induced HA secretion. Antigen-specific immunotherapies using TSHR peptides to reduce serum TSHR antibodies are being developed also. These TSHR-targeted strategies also have the potential to treat both GH and TED with the same drug. We propose that combination therapy targeting TSHR and IGF$1 R$ may be an effective and better tolerated treatment strategy for TED.

(c) 2020 European Thyroid Association Published by S. Karger AG, Basel

\section{TSH/IGF-1 Receptor Crosstalk in Graves' Orbital Fibroblasts in Primary Culture}

The discovery of thyroid-stimulating hormone receptor (TSHR) expression in orbital fat tissue and extra-ocular muscles [1] suggested that TSHR is one of the primary autoantigens in orbital tissue in patients with thyroid eye disease (TED)/Graves' orbitopathy (GO). The
Marvin C. Gershengorn

50 South Dr. Building 50, Room 4134 Bethesda, MD 20892 (USA)

MarvinG@intra.niddk.nih.gov 


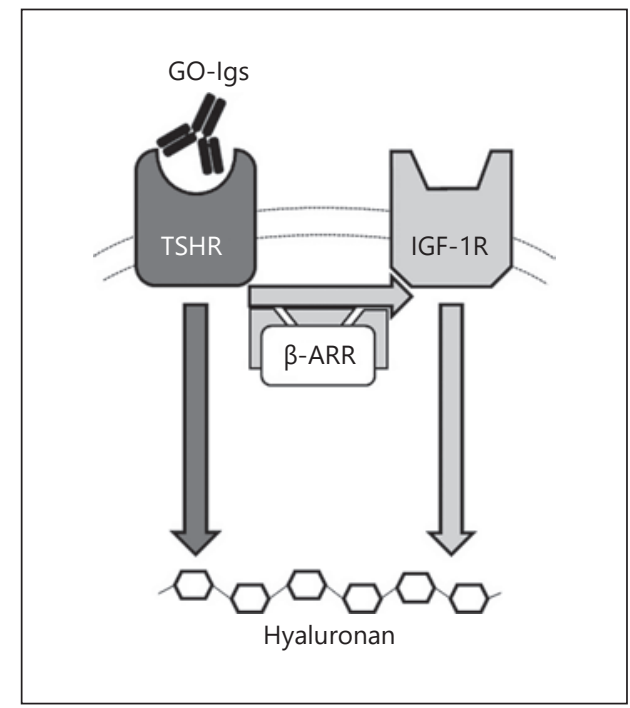

Fig. 1. Model of TSHR/IGF-1R crosstalk in TED pathogenesis. Activation of TSHR by GO-Igs in GOFs activates two signal transduction pathways - one that is independent of IGF-1R (dark gray arrow) and another that is dependent on IGF-1R (light gray arrows). TSHR and IGF-1R are in close proximity within a signalosome for which $\beta$-arrestin 1 ( $\beta$-ARR) acts as a scaffold. TSHR- and IGF-1Rmediated pathways combine through crosstalk, leading to a more robust stimulation of HA secretion. Modified from Krieger et al. $[7,41]$.

insulin-like growth factor 1 (IGF-1) receptor (IGF-1R) has been identified as another important player in the pathogenesis of TED. Functional interactions between TSHRs and IGF-1Rs have been shown in orbital fibroblasts from patients with Graves' disease (GOFs) in vitro [2-8]. Stimulated secretion of hyaluronan (hyaluronic acid, HA) has often been measured as a biologic response because increased HA secretion in the orbit is a major component of the pathophysiology of TED. Functionally important TSHR/IGF-1R crosstalk in GOFs was initially demonstrated by showing that simultaneous activation by TSH and IGF-1 synergistically increased HA secretion by GOFs [6]. Synergistic increases in both potency and efficacy of TSH in the presence of IGF-1 were shown. Additional evidence in support of the occurrence of TSHR/ IGF-1R crosstalk in GOFs was provided by the following observations: (1) a small molecule IGF-1R inhibitor, linsitinib, inhibited HA stimulation by TSH [6]; (2) the high-potency phase of the biphasic dose response of HA secretion stimulated by a monoclonal TSHR-stimulating antibody (TSAb) M22, which was derived from a patient with Graves' hyperthyroidism (GH) [9] and does not bind to IGF-1R [10], was inhibited by the IGF-1R antagonist linsitinib [6]; (3) stimulation by M22 and immuno- globulins purified from the sera of Graves' disease (GD) patients with eye disease (GO-Igs) was inhibited by some, but not all, IGF-1R-blocking antibodies (see below) $[4,5$, $7,11]$; and (4) TSHR and IGF-1R were shown to be in close proximity to each other scaffolded by $\beta$-arrestin 1 , which was required for TSHR/IGF-1R crosstalk [12].

These findings are summarized in our proposed model of TSHR/IGF-1R crosstalk which is illustrated in Figure 1. GO-Igs bind directly to and activate the TSHR in orbital fibroblasts. TSHR and IGF-1R are in close proximity within a signalosome for which $\beta$-arrestin 1 acts as a scaffold. Two pathways lead to HA secretion, a TSHR-dependent, IGF-1R-independent pathway and a TSHR-dependent, IGF-1R-dependent pathway. We propose that the activated TSHR engages the IGF-1R in a signalosome which induces the synergistic increase in HA secretion. As TSHR and IGF-1R signal in concert when initiating the signaling cascades leading to HA secretion in the pathogenesis of TED, it may be desirable to target both receptors with therapeutic interventions.

\section{IGF-1R-Blocking Antibodies}

IGF-1R-blocking antibodies, such as AF305, 1H7, and teprotumumab, have been studied to gain insight into a potential role for IGF-1R in mediating stimulation of HA secretion by GO-Igs $[5,7,13]$. We first compared the effects of AF305 and $1 \mathrm{H} 7$ on HA secretion stimulated by IGF-1 and M22 [7]. As expected, AF305 and 1H7 markedly inhibited HA secretion stimulated by IGF-1 (Fig. 2a). In contrast, AF305 had no effect on stimulation by M22 whereas $1 \mathrm{H} 7$ partially inhibited M22 stimulation. Teprotumumab has been shown to exhibit inhibition similar to that of $1 \mathrm{H} 7$ [5]. We conclude that AF305 acts only by inhibiting the IGF-1R while $1 \mathrm{H} 7$ in addition inhibits TSHR/IGF-1R crosstalk through a yet incompletely understood mechanism. The monoclonal antibody M22 might not represent GOF stimulation by all polyclonal antibodies found in sera of Graves' patients. Therefore, we tested purified GO-Igs from patients with eye disease. To keep the TSHR antibody concentration as close as possible to the original patient's sample, we reconstituted the samples after purification to their original volume. The purification of serum samples did not change the heterogeneous mixture of stimulatory and blocking TSHR antibodies since all serum Igs were purified. 1H7 was shown to inhibit HA secretion by 57 GO-Igs while AF305 had no effect on GO-Ig stimulation of HA secretion (Fig. 2b) [7]. We suggest that teprotumumab inhib-
Neumann/Krieger/Gershengorn 


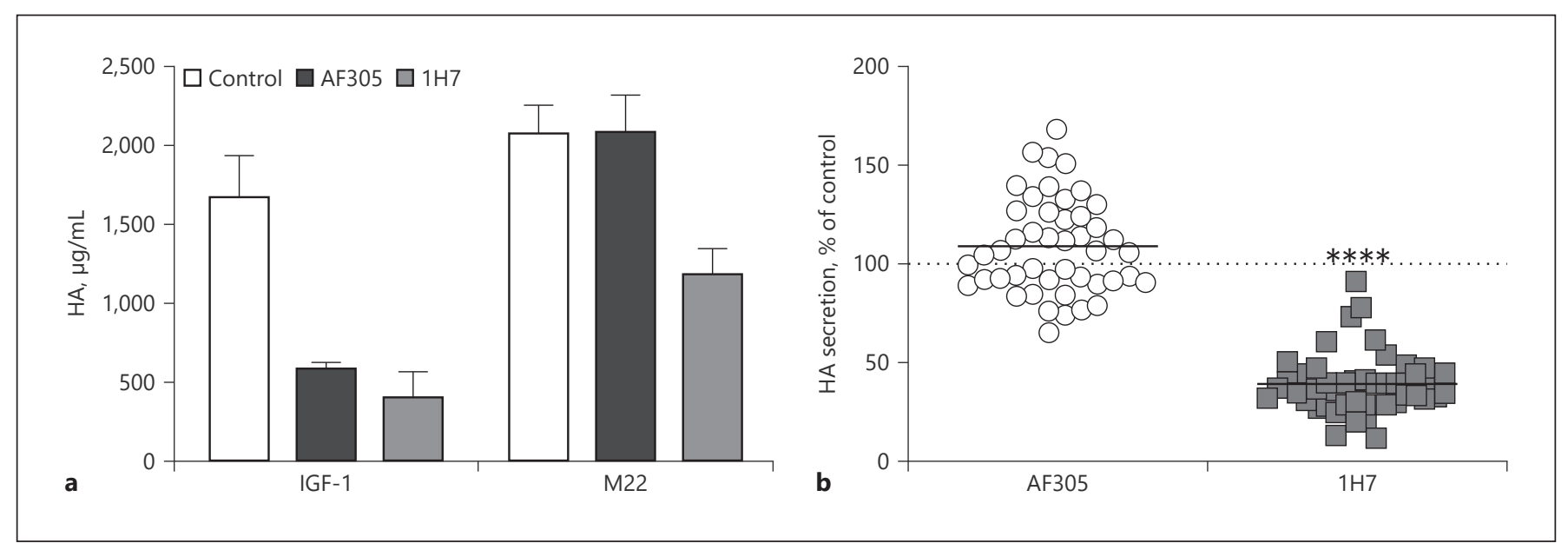

Fig. 2. 1H7, but not AF305, inhibits M22 and GO-Ig stimulation of HA secretion. a GOFs were stimulated by maximally effective doses of IGF-1 or the TSAb M22 in the absence (control) or presence of the IGF-1R-blocking antibodies AF305 or 1H7, and HA secretion was measured after 5 days. Both AF305 and $1 \mathrm{H} 7$ inhibited HA secretion stimulated by IGF-1 but only 1H7, not AF305, inhibited stimulation by M22. Modified from Krieger et al. [7]. b GOFs were stimulated by purified GO-Igs from 57 patients in

its HA secretion via a mechanism similar to that of $1 \mathrm{H} 7$, that is, by inhibiting TSHR/IGF-1R crosstalk. Of note, teprotumumab has been shown to be effective in the treatment of patients with TED [14-16] and has recently been approved by the United State Food and Drug Administration (FDA) (TEPEZZA, https://www.fda.gov/ news-events/press-announcements/fda-approves-firsttreatment-thyroid-eye-disease).

\section{TSHR-Targeted Treatments}

TSAbs found in patients with GH and TED directly activate TSHR. Therefore, targeting the TSHR for therapeutic intervention would have the advantage to treat $\mathrm{GH}$ and TED with the same drug. It is not yet understood whether the same TSAbs activate TSHRs in the thyroid and in the eye, and whether the signaling cascades are similar or different in these tissues. It is conceivable that different antibody populations might target TSHRs in the thyroid or the retro-orbital tissue as there are different disease phenotypes. Patients can present with GH with or without TED. Moreover, TED can be observed in TSAbpositive patients with autoimmune thyroiditis or in the absence of thyroid disfunction (euthyroid TED). The ability to shed light on these questions will also guide the the absence (not shown) or presence of the IGF-1R-blocking antibody AF305 or $1 \mathrm{H} 7$, and HA secretion was measured after 5 days. The data are presented as percent of control in the absence of AF305 or $1 \mathrm{H} 7$. In contrast to AF305, $1 \mathrm{H} 7$ decreased HA secretion by all GO-Ig preparations (mean inhibition of $67 \%$ ) by inhibiting TSHR/IGF-1R crosstalk. ${ }^{* * * *} p<0.0001$ versus GO-Ig control by Student's $t$ test [7].

development of therapies that target TSHR as well as TSHR/IGF-1R crosstalk.

Studies with purified GO-Igs have underlined that TSHR/IGF-1R crosstalk is initiated by binding of GO-Igs to TSHRs [7]. Considering the IGF-1R-independent component of GO-Ig-induced HA secretion, it is conceivable that TSHR antagonists may be more efficient in blocking the effect of stimulating TSHR antibodies. Moreover, the effects of TSHR antagonists will function over the entire concentration range of GO-Igs whereas IGF-1R antagonists may function only over the range of GO-Ig concentrations that activate TSHR/IGF-1R crosstalk.

Monoclonal antibodies directed at TSHR have been studied as antagonists of TSHR activation by TSHRbinding agonists [17-19]. Blocking antibodies inhibit activation of TSHR by blocking the binding of GO-Igs to the extracellular domain of TSHR. A human monoclonal TSHR-blocking antibody, K1-70 [20], is being studied as a potential treatment for GD [21]. It was shown to be effective in vivo causing biochemical hypothyroidism in untreated and M22-treated rats [22], and it has recently undergone a preclinical toxicology study in rats and cynomolgus monkeys [23]. When K1-70 was administered to a female patient with GD and severe TED, an improvement in the patient's clinical activity score and exophthalmos was observed [24]. K1-70 is a promising treatment 


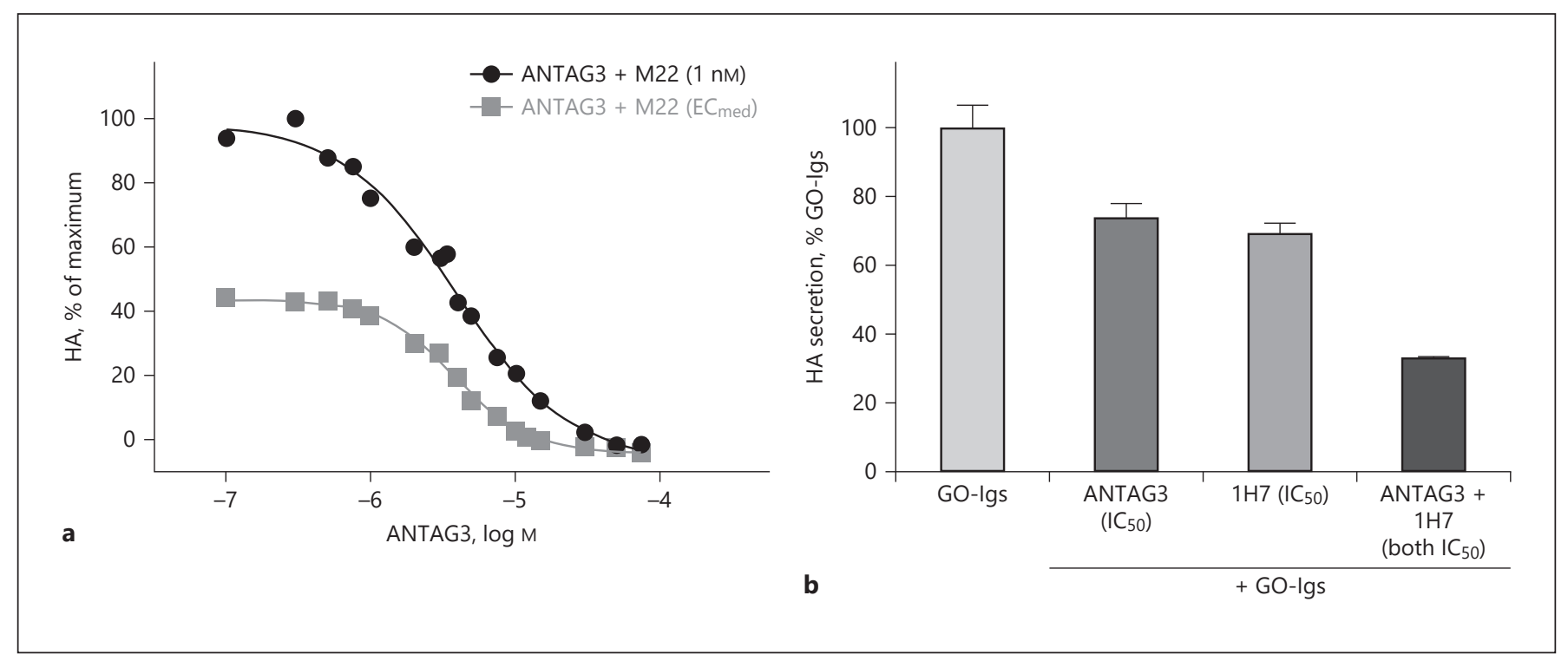

Fig. 3. Combination of ANTAG3 and $1 \mathrm{H} 7$ additively inhibits GOIg stimulation. a ANTAG3 is a fully efficacious TSHR antagonist for M22-induced HA secretion in GOFs regardless of M22 concentration. Cultured GOFs were stimulated with $1 \mathrm{nM} \mathrm{M} 22$, which is a maximally effective dose, or the half-maximally effective dose (EC $\left.\mathrm{E}_{\mathrm{med}}\right)$ of $\mathrm{M} 22$, and cotreated with increasing doses of the small molecule TSHR antagonist ANTAG3 for 4 days. Total HA secretion was measured in the culture media. Data represent the mean of two donor cell strains plotted as percent HA levels relative to the $1 \mathrm{nM} \mathrm{M} 22$ maximal response [11]. b Combination treatment with

option and currently in phase I clinical trials (https:// Clinical Trials.gov/show/NCT02904330) [25].

Recently, antigen-specific immunotherapies have been developed as an attractive treatment option for GD $[26,27]$. Cyclic peptides that mimic one of the cylindrical loops of the leucine-rich repeat domain in the extracellular domain of TSHR have been studied and act via an immune-mediated mechanism to treat GH and TED [28, 29]. The hypothesis behind this approach is to generate a TSHR ectodomain immune hyposensitization that would specifically limit anti-TSHR autoantibody production [26]. This approach was shown to decrease disease manifestations in a mouse model of GD [29].

Alternatively, T-cell epitope-derived linear peptides based on the sequence of the TSHR have been identified using immunized HLA-DR3 transgenic mice, which induce tolerance towards TSHR [30]. A first-in-human antigen-specific immunotherapy was conducted through immunization with TSHR peptide ATX-GD-59 [27]. Ten patients with mild to moderate GH received treatment with ATX-GD-59 for 18 weeks. The serum TSHR antibody concentration was reduced after treatment and cor-
TSHR and IGF-1R antagonists on HA stimulation by purified GOIgs efficiently inhibits TSHR/IGF-1R crosstalk. Cultured GOFs were stimulated with GO-Igs purified from the sera of 6 different donors in the absence (not shown) or presence of the half-maximally effective inhibitory concentration $\left(\mathrm{IC}_{50}\right)$ of the TSHR antagonist ANTAG3 or the IGF-1R-blocking antibody $1 \mathrm{H} 7$ or both. HA secretion was measured after 5 days. Inhibition with an $\mathrm{IC}_{50}$ dose of each antagonist separately is not complete; however, the combination of both antagonists at $\mathrm{IC}_{50}$ doses was additive [11].

related with changes in serum free thyroid hormone levels [27]. This study suggests potential for ATX-GD-59 as treatment for $\mathrm{GH}$, but it still must be determined whether it will also be effective for the treatment of TED.

We [31-34] and others [35-38] continue in attempts to develop small molecule, drug-like ligands as antagonists of TSHR signaling. Small molecule compounds have the advantages of oral bioavailability, a short to long half-life which broadens potential clinical applications and bioavailability, favorable pharmacokinetics, and lower production costs, which supports further development of this drug type. Small molecule antagonists bind to TSHRs at sites different from TSH; TSH binds to the extracellular domain of TSHR whereas these antagonists bind within the transmembrane domain. These antagonists do not inhibit GOIg binding to the extracellular TSHR domain but inhibit signal transduction from the extracellular to the intercellular domain by binding to the transmembrane domain of the receptor. An important advantage of these small organic molecule TSHR antagonists is their oral availability that would allow easier administration outside the clinic compared to antibodies that must be administered parenterally. 
Small molecule TSHR antagonists have been extensively reviewed recently [39], and therefore, we will focus on selected compounds. Latif et al. [37] developed an indole-thiazole derivative named VA-K-14. This small molecule antagonist inhibited TSHR stimulation by GD patient sera and monoclonal-stimulating TSHR antibodies. VA-K-14 showed only slight selectivity towards the TSHR compared to luteinizing hormone receptor or follicle-stimulating hormone receptor, which are the receptors with the highest homology to TSHR within the seven transmembrane domain [40], in which the small molecule TSHR antagonists bind. Recently, Marcinkowski et al [38]. developed the TSHR antagonist S37. Its active enantiomer S37a is highly selective for the TSHR and does not antagonize luteinizing hormone receptor or folliclestimulating hormone receptor signaling. S37a inhibits cAMP production induced by TSH, the TSAb M22, and by polyclonal TSAbs from sera of patients with TED. Pharmacokinetic studies showed good bioavailability and tolerance of S37a in mice but an inhibitory effect of this antagonist in an in vivo model of GD or TED has yet to be demonstrated.

We have developed ANTAG3 (NCGC00242364) [33], which is also selective for TSHR since it does not inhibit the signaling by luteinizing hormone receptor or folliclestimulating hormone receptor. ANTAG3 inhibits TSHR activation in mice in vivo [33]. This selective antagonist has been shown to be effective in inhibiting TSHR-mediated responses in an in vivo mouse model of thyroid gland stimulation by endogenous TSH. ANTAG3 inhibited the elevation in serum free thyroxine. Furthermore, ANTAG3 inhibited the increase in thyroperoxidase and sodium-iodide symporter mRNA expression caused by continuous administration of thyrotropin-releasing hormone [33]. This was the first demonstration in an animal model of a TSHR-selective, small molecule antagonist that is effective in inhibiting thyroid gland stimulation suggesting that a small molecule TSHR antagonist like ANTAG3 could be used to treat GH. We have also shown that ANTAG3 at maximally effective doses can fully inhibit M22 stimulation of HA secretion by cultured GOFs indicating additional potential for the treatment of TED (Fig. 3a) [11].

\section{Combination Therapy with TSHR and IGF-1R Antagonists}

As described above, TSHR/IGF-1R crosstalk allows for two pathways initiated by GO-Ig activation of TSHR, one IGF-1R-dependent and another IGF-1R-independent, to

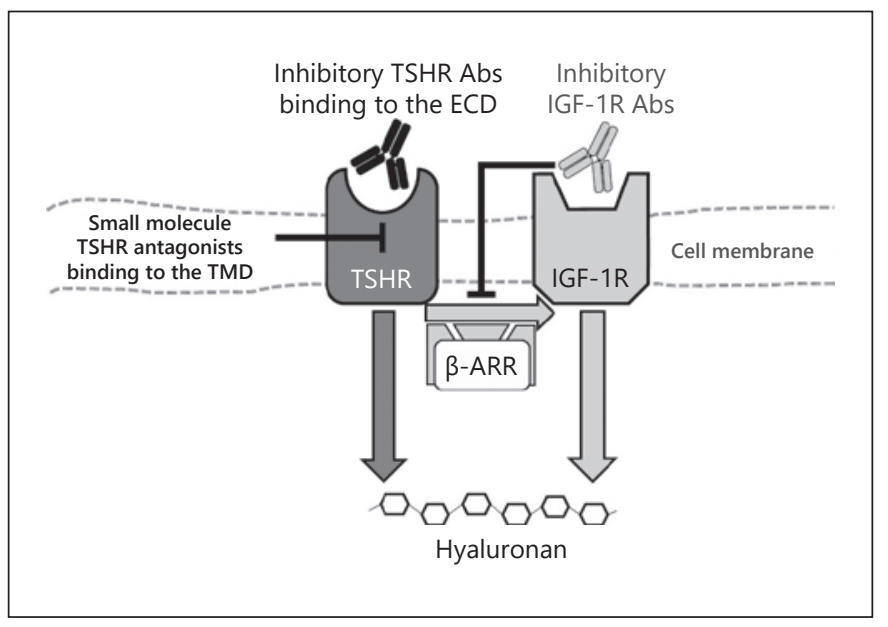

Fig. 4. Model of inhibition of TSHR/IGF-1R crosstalk by targeting TSHR and/or IGF-1R. Activation of the TSHR by GO-Igs stimulates two signal transduction pathways - one that is independent of IGF-1R (dark gray arrow) and another that is dependent on IGF-1R, which is the crosstalk pathway (light gray arrows). The activated TSHR engages the IGF-1R in a signalosome that is scaffolded by $\beta$-arrestin 1 ( $\beta$-ARR), and this crosstalk induces the synergistic increase in HA secretion. As TSHR and IGF-1R signal in concert in the pathogenesis of TED, therapeutic interventions that target both receptors may be desirable. In contrast to antigen-specific immunotherapies which use peptides of sequences in the extracellular domain of the TSHR, inhibitory TSHR antibodies (Abs) and small molecule TSHR antagonists directly target the TSHR and inhibit signaling. TSHR-blocking antibodies inhibit TSHR activation by binding to the extracelluar domain (ECD) of the TSHR thereby inhibiting GO-Igs from binding. Drug-like, small molecule TSHR antagonists bind to allosteric sites in the transmembrane domain (TMD) of TSHR and inhibit activation by hindering signal transduction through the TSHR from the extracellular to the intracellular domain which subsequently inhibits interaction of the receptor with $G$ proteins and other intracellular signaling molecules. IGF-1R-inhibiting antibodies, like $1 \mathrm{H} 7$ and teprotumum$\mathrm{ab}$, bind to IGF-1R and inhibit IGF-1R crosstalk with TSHR. It is conceivable that a combination therapy with TSHR and IGF-1R antagonists may have therapeutic benefits as it could minimize drug side effects due to dose reduction and may compensate for any loss of anti-IGF-1R efficacy.

lead to maximal stimulation of HA secretion by GOFs. We showed that the small molecule, drug-like antagonist ANTAG3 was more effective in inhibiting GO-Ig-stimulated HA secretion than the IGF-1R-blocking antibody 1H7 [11]. This effect is expected as ANTAG3 inhibited both IGF-1R-dependent and IGF-1R-independent pathways whereas $1 \mathrm{H} 7$ was only partially inhibitory as it inhibited only the IGF-1R-dependent pathway. Based on these findings, we reasoned that we could increase the therapeutic index for this effect by lowering the doses of both antagonists when used in combination. In combina- 
tion, these antagonists exhibited Loewe additivity within the IGF1R-dependent component of the M22 biphasic concentration response in GOFs [11]. Similar additivity was observed in GOFs activated by GO-Igs from patients' sera (Fig. 3b). We suggest that combination therapy may be an effective strategy for dose reduction and/or may compensate for any loss of anti-IGF-1R efficacy (Fig. 4) [11].

\section{Concluding Remarks}

We acknowledge the exciting findings of the humanized monoclonal anti-IGF-1R-blocking antibody teprotumumab as an effective treatment of TED [14-16] and its current approval by the FDA. However, there are drawbacks to its use. As a therapeutic antibody, it must be administered parenterally necessitating a clinic visit. Also, as it only interferes with the IGF-1R-dependent pathway, it may lose efficacy in patients with high titers of GO-Igs that primarily activate the IGF-1R-independent pathway. TSHR-blocking antibodies will have to be administered parenterally also but should be more effective than teprotumumab as they will inhibit both IGF-1R-dependent and IGF-1R-independent pathways. As do blocking anti- bodies, small molecule, drug-like TSHR antagonists will inhibit both pathways. More importantly, they have the important advantage of oral administration without the need to attend a clinic. Thus, we and others continue to pursue the discovery/development of a small molecule, drug-like TSHR antagonist.

\section{Disclosure Statement}

Christine Carag Krieger, Susanne Neumann, and Marvin Carl Gershengorn have filed a patent pertaining to drug combinations targeting TSHR and IGF-1R (application No.: KS Ref. 4239-9389701, E-No. E-284-2008/1).

\section{Funding Sources}

This research was supported by the intramural research program (Z01 DK011006) of the National Institutes of Health.

\section{Author Contributions}

All authors have contributed significantly to the work, have read the manuscript, attest to the validity and legitimacy of the data and its interpretation, and agree to its submission.

\section{References}

1 Feliciello A, Porcellini A, Ciullo I, Bonavolontà G, Avvedimento EV, Fenzi G. Expression of thyrotropin-receptor mRNA in healthy and Graves' disease retro-orbital tissue. Lancet. 1993 Aug;342(8867):337-8.

2 Tsui S, Naik V, Hoa N, Hwang CJ, Afifiyan NF, Sinha Hikim A, et al. Evidence for an association between thyroid-stimulating hormone and insulin-like growth factor 1 receptors: a tale of two antigens implicated in Graves' disease. J Immunol. 2008 Sep;181(6): 4397-405.

3 Hoa N, Tsui S, Afifiyan NF, Sinha Hikim A, Li B, Douglas RS, et al. Nuclear targeting of IGF-1 receptor in orbital fibroblasts from Graves' disease: apparent role of ADAM17. PLoS One. 2012;7(4):e34173.

4 Kumar S, Iyer S, Bauer H, Coenen M, Bahn RS. A stimulatory thyrotropin receptor antibody enhances hyaluronic acid synthesis in graves' orbital fibroblasts: inhibition by an IGF-I receptor blocking antibody. J Clin Endocrinol Metab. 2012 May;97(5):1681-7.

5 Chen H, Mester T, Raychaudhuri N, Kauh CY, Gupta S, Smith TJ, et al. Teprotumumab, an IGF-1R blocking monoclonal antibody inhibits TSH and IGF-1 action in fibrocytes. J Clin Endocrinol Metab. 2014 Sep; 99(9):E1635-40.
6 Krieger CC, Neumann S, Place RF, MarcusSamuels B, Gershengorn MC. Bidirectional TSH and IGF-1 receptor cross talk mediates stimulation of hyaluronan secretion by Graves' disease immunoglobins. J Clin Endocrinol Metab. 2015 Mar;100(3):1071-7.

7 Krieger CC, Place RF, Bevilacqua C, MarcusSamuels B, Abel BS, Skarulis MC, et al. TSH/ IGF-1 Receptor Cross Talk in Graves' Ophthalmopathy Pathogenesis. J Clin Endocrinol Metab. 2016 Jun;101(6):2340-7.

8 Morgan SJ, Neumann S, Marcus-Samuels B, Gershengorn MC. Thyrotropin and InsulinLike Growth Factor 1 Receptor Crosstalk Upregulates Sodium-Iodide Symporter Expression in Primary Cultures of Human Thyrocytes. Thyroid. 2016 Dec;26(12):1794-803.

9 Sanders J, Evans M, Premawardhana LD, Depraetere $\mathrm{H}$, Jeffreys J, Richards $\mathrm{T}$, et al. Human monoclonal thyroid stimulating autoantibody. Lancet. 2003 Jul;362(9378):126-8.

10 Krieger CC, Neumann S, Marcus-Samuels B, Gershengorn MC. TSHR/IGF-1R Cross-Talk, Not IGF-1R Stimulating Antibodies, Mediates Graves' Ophthalmopathy Pathogenesis. Thyroid. 2017 May;27(5):746-7.
11 Place RF, Krieger CC, Neumann S, Gershengorn MC. Inhibiting thyrotropin/insulin-like growth factor 1 receptor crosstalk to treat Graves' ophthalmopathy: studies in orbital fibroblasts in vitro. Br J Pharmacol. 2017 Feb; 174(4):328-40.

12 Krieger CC, Boutin A, Jang D, Morgan SJ, Banga JP, Kahaly GJ, et al. Arrestin- $\beta-1$ Physically Scaffolds TSH and IGF1 Receptors to Enable Crosstalk. Endocrinology. 2019 Jun; 160(6):1468-79.

13 Chen H, Shan SJ, Mester T, Wei YH, Douglas RS. TSH-Mediated TNF $\alpha$ Production in $\mathrm{Hu}-$ man Fibrocytes Is Inhibited by Teprotumumab, an IGF-1R Antagonist. PLoS One. 2015 Jun;10(6):e0130322.

14 Smith TJ, Kahaly GJ, Ezra DG, Fleming JC, Dailey RA, Tang RA, et al. Teprotumumab for Thyroid-Associated Ophthalmopathy. N Engl J Med. 2017 May;376(18):1748-61.

15 Douglas RS, Kahaly GJ, Patel A, Sile S, Thompson EH, Perdok R, et al. Teprotumumab for the Treatment of Active Thyroid Eye Disease. N Engl J Med. 2020 Jan;382(4):341-52.

16 Slentz DH, Nelson CC, Smith TJ. Teprotumumab: a novel therapeutic monoclonal antibody for thyroid-associated ophthalmopathy. Expert Opin Investig Drugs. 2020 Jul; 29(7):645-9. 
17 Chen CR, McLachlan SM, Rapoport B. Suppression of thyrotropin receptor constitutive activity by a monoclonal antibody with inverse agonist activity. Endocrinology. 2007 May;148(5):2375-82.

18 Sanders J, Miguel RN, Furmaniak J, Smith BR TSH receptor monoclonal antibodies with agonist, antagonist, and inverse agonist activities. Methods Enzymol. 2010;485:393-420.

19 Majumdar R, Railkar R, Dighe RR. The antibodies against the computationally designed mimic of the glycoprotein hormone receptor transmembrane domain provide insights into receptor activation and suppress the constitutively activated receptor mutants. J Biol Chem. 2012 Oct;287(41):34514-32.

20 Evans M, Sanders J, Tagami T, Sanders P, Young S, Roberts E, et al. Monoclonal autoantibodies to the TSH receptor, one with stimulating activity and one with blocking activity, obtained from the same blood sample. Clin Endocrinol (Oxf). 2010 Sep;73(3):40412.

21 Furmaniak J, Sanders J, Rees Smith B. Blocking type TSH receptor antibodies. Auto Immun Highlights. 2012 Mar;4(1):11-26.

22 Furmaniak J, Sanders J, Young S, Kabelis K, Sanders P, Evans M, et al. In vivo effects of a human thyroid-stimulating monoclonal autoantibody (M22) and a human thyroidblocking autoantibody (K1-70). Auto Immun Highlights. 2011 Sep;3(1):19-25.

23 Furmaniak J, Sanders J, Clark J, Wilmot J, Sanders P, Li Y, et al. Preclinical studies on the toxicology, pharmacokinetics and safety of K1-70TM a human monoclonal autoantibody to the TSH receptor with TSH antagonist activity. Auto Immun Highlights. 2019 Nov; 10(1):11.

24 Furmaniak J, Ryder MM, Castro MR, Wentworth M, Algeciras A, Morris JC, et al. Blocking the TSH receptor with the human monoclonal autoantibody K1-70 (TM) improves Graves' ophthalmopathy and aids control of advanced follicular thyroid carcinoma - results of long-term treatment under the first in human single patient expanded use therapy. Eur Thyroid J. 2018 Aug;7:22.

25 Singh D. K1-70 - a study in subjects with Graves' disease. 2016. Available from: http:// clinicaltrials.gov/ct2/show/NCT02904330

26 Ungerer M, Fabbender J, Holthoff HP. Antigen-specific therapy of Graves' disease and orbitopathy by induction of tolerance. Front Biosci. 2018 Jun;23(11):2044-52.

27 Pearce SH, Dayan C, Wraith DC, Barrell K, Olive N, Jansson L, et al. Antigen-Specific Immunotherapy with Thyrotropin Receptor Peptides in Graves' Hyperthyroidism: A Phase I Study. Thyroid. 2019 Jul;29(7):100311.

28 Holthoff HP, Li Z, Faßbender J, Reimann A, Adler K, Münch G, et al. Cyclic Peptides for Effective Treatment in a Long-Term Model of Graves Disease and Orbitopathy in Female Mice. Endocrinology. 2017 Jul;158(7):237690.

29 Faßbender J, Holthoff HP, Li Z, Ungerer M. Therapeutic Effects of Short Cyclic and Combined Epitope Peptides in a Long-Term Model of Graves' Disease and Orbitopathy. Thyroid. 2019 Feb;29(2):258-67.

30 Jansson L, Vrolix K, Jahraus A, Martin KF, Wraith DC. Immunotherapy With Apitopes Blocks the Immune Response to TSH Receptor in HLA-DR Transgenic Mice. Endocrinology. 2018 Sep;159(9):3446-57.

31 Neumann S, Pope A, Geras-Raaka E, Raaka BM, Bahn RS, Gershengorn MC. A drug-like antagonist inhibits thyrotropin receptor-mediated stimulation of cAMP production in Graves' orbital fibroblasts. Thyroid. 2012 Aug;22(8):839-43.

32 Turcu AF, Kumar S, Neumann S, Coenen M, Iyer S, Chiriboga $\mathrm{P}$, et al. A small molecule antagonist inhibits thyrotropin receptor antibody-induced orbital fibroblast functions involved in the pathogenesis of Graves ophthalmopathy. J Clin Endocrinol Metab. 2013 May; 98(5):2153-9.

33 Neumann S, Nir EA, Eliseeva E, Huang W, Marugan J, Xiao J, et al. A selective TSH re- ceptor antagonist inhibits stimulation of thyroid function in female mice. Endocrinology. 2014 Jan;155(1):310-4.

34 Neumann S, Place RF, Krieger CC, Gershengorn MC. Future Prospects for the Treatment of Graves' Hyperthyroidism and Eye Disease. Horm Metab Res. 2015 Sep;47(10) 789-96.

35 van Koppen CJ, de Gooyer ME, Karstens WJ, Plate R, Conti PG, van Achterberg TA, et al. Mechanism of action of a nanomolar potent, allosteric antagonist of the thyroid-stimulating hormone receptor. Br J Pharmacol. 2012 Apr;165(7):2314-24

36 van Zeijl CJ, van Koppen CJ, Surovtseva OV, de Gooyer ME, Plate R, Conti P, et al. Complete inhibition of rhTSH-, Graves' disease IgG-, and M22-induced cAMP production in differentiated orbital fibroblasts by a low-molecular-weight TSHR antagonist. J Clin Endocrinol Metab. 2012 May;97(5):E781-5.

37 Latif R, Realubit RB, Karan C, Mezei M, Davies TF. TSH receptor signaling abrogation by a novel small molecule. Front Endocrinol (Lausanne). 2016 Sep;7:130.

38 Marcinkowski P, Hoyer I, Specker E, Furkert J, Rutz C, Neuenschwander M, et al. A New Highly Thyrotropin Receptor-Selective Small-Molecule Antagonist with Potential for the Treatment of Graves' Orbitopathy. Thyroid. 2019 Jan;29(1):111-23.

39 Krause G, Marcinkowski P. Intervention Strategies into Glycoprotein Hormone Receptors for Modulating (Mal-)function, with Special Emphasis on the TSH Receptor. Horm Metab Res. 2018 Dec;50(12):894907

40 Kleinau G, Krause G. Thyrotropin and homologous glycoprotein hormone receptors: structural and functional aspects of extracellular signaling mechanisms. Endocr Rev. 2009 Apr;30(2):133-51.

41 Krieger CC, Neumann S, Gershengorn MC TSH/IGF1 receptor crosstalk: mechanism and clinical implications. Pharmacol Ther. 2020 May;209:107502. 